\title{
Cyclic Scheduling of Pulp Digesters with Integrated Heating Tasks
}

\author{
Pedro M. Castro, ${ }^{* \dagger}$ Djêide Rodrigues, ${ }^{\dagger, \ddagger}$ and Henrique A. Matos ${ }^{\ddagger}$ \\ ${ }^{\dagger}$ Laboratório Nacional de Energia e Geologia, 1649-038 Lisboa, Portugal \\ ${ }^{\ddagger}$ Centro de Processos Químicos, DEQ Instituto Superior Técnico, 1049-001 Lisboa, Portugal
}

ABSTRACT: This paper addresses a multistage batch plant scheduling problem under energy constraints. These reflect the limited availability of a thermal heating utility that is shared among parallel digesters of different capacities for the production of pulp. Depending on the processing sequence, more or less steam will be available for a given digester, which will affect the duration of its heating stage and the overall cycle time. Such integrated heating tasks resemble direct heat integration, which has been addressed through models based on generic frameworks for process representation (e.g., State-Task Network, ResourceTask Network, State-Sequence Network) and relying on a single time grid, either discrete or continuous. A new multiple time grid continuous-time model is now proposed where the complex energy constraints are derived from the higher level modeling framework that is Generalized Disjunctive Programming. The results show a considerable better performance compared to RTN discrete and continuous-time formulations, due to a substantially lower integrality gap and model size.

\section{INTRODUCTION}

Despite the many developments that have occurred in the past 25 years, it is often difficult to predict the most efficient scheduling model for a particular problem. As highlighted in the recent review paper by Harjunkoski et al., ${ }^{1}$ general STN/ $\mathrm{RTN}^{2,3}$ models for network production environments come really close to addressing the different types of constraints that can be encountered at a process plant. However, special features such as a multistage topology can greatly simplify the mathematical formulation, which in turn increases the likelihood for the solution of large-scale industrial problems. Ideally, one should take advantage of the latter bearing in mind that they have been tuned to essentially handle constraints on discrete resources (e.g., equipment units), transfer of material between stages, and release/due dates. Models for sequential environments also rely on a continuous-time representation, which makes them more suitable to handle variable processing times.

Important constraints to be handled by scheduling models are those related to energy use. The topic of industrial demand side management, ${ }^{4,5}$ linked to the smart grid concept, has raised interest in models that can incorporate hourly changing electricity prices so as to minimize the total energy cost. Complex energy constraints can also be found in models allowing for heat integration in batch plants, ${ }^{6-8}$ where heat sources and sinks need to overlap in time.

Papageorgiou et al. ${ }^{6}$ addressed both direct and indirect heat integration with a discrete-time STN formulation. The superstructure for the example problem considered included alternative paths for reaction and distillation so as to handle stand-alone as well as heat-integrated operations. The more complex case of indirect heat integration, which involves a heat transfer medium to allow for energy storage and hence a more flexible operation, required a detailed characterization of mass and energy balances for the heat transfer medium used for implementing the integration. It introduces nonlinearities that change the nature of the formulation from a mixed-integer linear (MILP) to a nonconvex mixed-integer nonlinear program (MINLP). Focusing on direct heat integration, Majozi $^{7}$ proposed a continuous-time State-Sequence-Network (SSN) formulation to handle cases where (i) energy requirements depend on the batch size ${ }^{6}$ (allowed to vary), leading to a MINLP, and (ii) energy requirements are specified as parameters for fixed batch sizes, leading to a MILP. Working with the same problems, Chen and Chang ${ }^{8}$ extended a continuous-time RTN formulation for both short-term as well as cyclic operating modes.

Overall, the unified frameworks for process representation (STN, RTN, SSN) provide the necessary flexibility to model complex interactions between tasks or specific segments of tasks, which is the primary reason for their success in the Process Systems Engineering literature. They can be linked to either discrete- or continuous-time formulations, using one or multiple time grids, representing the top branch of Figure 1 (while not as explored, discrete-time formulations can also benefit from unit, state, and task specific time grids, as recently shown by Velez and Maravelias ${ }^{9}$ ). In particular, the heat integration formulations just described rely on a single time grid.

As highlighted by Grossmann and Trespalacios in a recent review paper, ${ }^{10}$ mixed-integer linear or nonlinear programming models are based on algebraic formulations that are not unique, leading to either solvable or nonsolvable problems. The Generalized Disjunctive Programming ${ }^{11}$ (GDP) higher-level modeling framework, by allowing the representation of constraints as algebraic equations, disjunctions, and logic propositions, makes the formulation process more intuitive and systematic, providing a structured way for systematically deriving models that exhibit strong continuous relaxations,

Special Issue: Jaime Cerdá Festschrift

Received: November 11, 2013

Revised: January 17, 2014

Accepted: January 28, 2014

Published: January 28, 2014 